\title{
Research on Initial Lightning Attachment Zone for Aircraft
}

\author{
Xiang-Ru Teng, Guang-Bin Liu, Zhi-Yong Yu, \\ Xin-Wu Zhuang \\ Xi’an Research Inst. of Hi-Tech \\ $\mathrm{Xi}$ ' an, China \\ tengxr_1989@163.com
}

\author{
Yu-Long Zhao \\ College of National Defense Information Science \\ Wuhan, China \\ wuzhangyuanlt@163.com
}

\begin{abstract}
By the use of dipole theory and "point effect" theory, electromagnetic field at any point in space, initial attachment features between lightning and aircraft and electric field intensity at "sharp" and "terminal" parts were researched theoretically. Conclusion was got: "sharp" and "terminal" parts are include in initial lightning attachment zone and points that are closer to lightning emitter, atmospheric equipotential surface or ground are most likely to become the import and export of lightning.
\end{abstract}

Keywords-dipole theory; "point effect”; electromagnetic field; initial attachment features; initial lightning attachment zone; import; export, lightning

\section{INTRODUCTION}

Nowadays, aircraft lightning protection design is mainly divided into six steps:

1) Determining lightning attachment zone;

2) Building lightning environment;

3) Finding out systems and components which are likely damaged by lightning;

4) Setting lightning protection standards for systems and components;

5) Making protection design for systems and components;

6) Confirming the rationality of the protection design by the use of test.

It can be found easily that determining lightning attachment zone is very important. Reasonable dividing method for $1 \mathrm{~B}, 2$ and 3 zones is presented by a large amount of researches, and the $1 \mathrm{~A}$ zone is their foundation, therefore, determining $1 \mathrm{~A}$ zone is the key step ${ }^{[1],[2],[3],[4]}$."Point effect" theory and dipole theory are used to research the dividing of $1 \mathrm{~A}$ zone in this paper.

\section{CALCULATING OF ELECTROMAGNETIC FIELD AT ANY POINT IN SPACE}

It is a condition for lightning taking place between cloud and aircraft that the electric field intensity at the some point on the aircraft surface reaches the breakdown electric field intensity (E) of air (about 3MV), it is a key to calculate electric field intensity of points on aircraft surface.

Cloud-to-ground lightning and clouds lightning are two forms of natural lightning, but the observation of clouds lightning's features is so hard that lightning features mainly refer to statistics and measured data of cloud-to-ground lightning ${ }^{[5],[6]}$. It is safe and dependable to use clouds lightning's features as the foundation of lightning protection design for the reason that the discharging strength of cloudto-ground lightning is far large than that of clouds lightning.

Building up lightning return stroke channel model showed as Figure $1^{[7],[8]}$

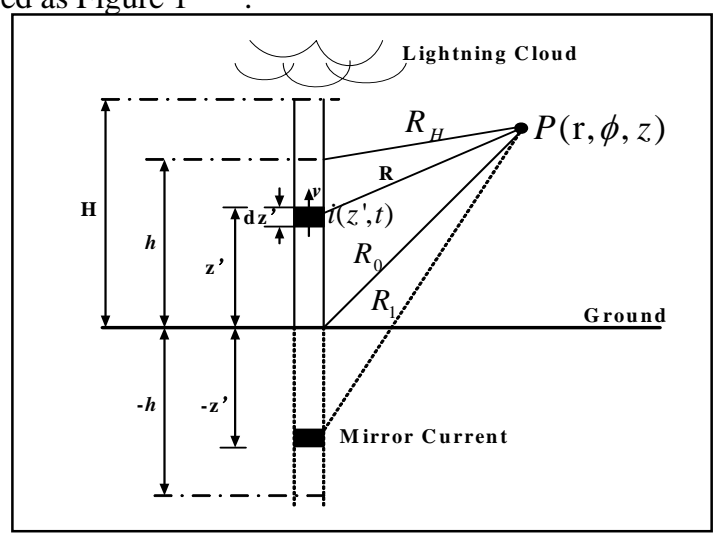

Figure 1 Lightning Return Sroke Channel Model

Equivalent lightning return stroke channel model as $\mathrm{H}-$ long vertical antenna that is perpendicular to the ground. $P(\mathrm{r}, \phi, z)$ stands for any space field point in electromagnetic field, lightning return stroke current transfers upward in uniform speed $v$ along the channel, $h$ stands for the height that the lead edge of return stroke current reaches, current higher than $h$ is 0 .

According to dipole theory, a micro-current unit $d z^{\prime}$ in the channel is selected as the research object, the height of which is z', and building up a current unit at the place of $-z^{\prime}$. The additional mirror current of discharging channel is $-\mathrm{H}$. a cylindrical coordinate system showed as Figure 2 is established based on what is narrated above to calculate the lightning electromagnetic field. 


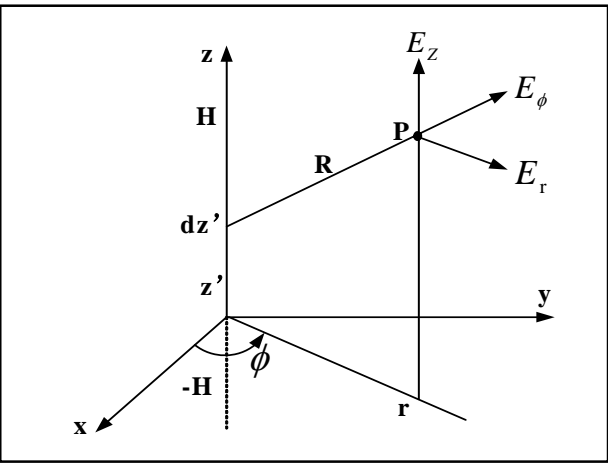

Figure 2 Lightning Electromagnetic Field Calculating Cylindrical Coordinate System

Maxwell equations of time-varying electromagnetic field are showed as below:

$$
\boldsymbol{B}=\mu_{0} \boldsymbol{H}
$$

where $\varepsilon_{0}$ is permittivity $(\mathrm{F} / \mathrm{m}), \mu_{0}$ is permeability $(\mathrm{H} / \mathrm{m})$, $\rho$ and $\boldsymbol{J}$ stand for density of electric charge and current respectively, $\boldsymbol{B}$ is magnetic induction (T), $\boldsymbol{E}$ is electric field intensity, $\boldsymbol{H}$ is magnetic field intensity $(\mathrm{A} / \mathrm{m})$, the speed of lightning radio wave is defined as $v_{1}\left(v_{1}=1 / \sqrt{\mu_{0} \varepsilon_{0}}\right)$, equal to speed of light in air, that is $2.998 \times 10^{8} \mathrm{~m} / \mathrm{s}$, lightning emitter is show as Figure 3.

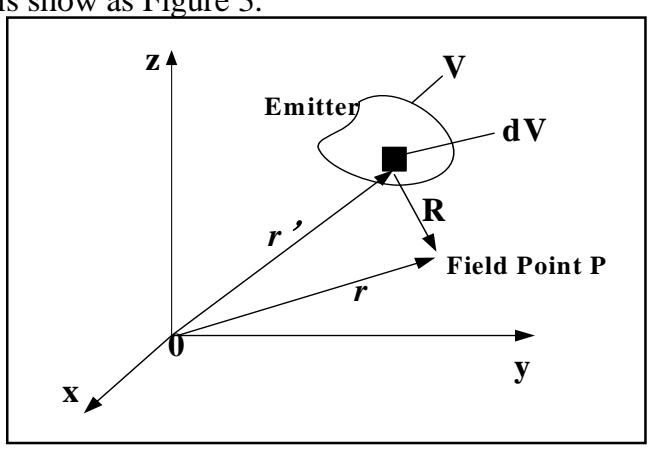

Figure 3 Lightning Emitter

We general use scalar quantity electric potential $\phi$ and magnetic vector potential $\boldsymbol{A}$ to take the place of $\boldsymbol{E}$ and $\boldsymbol{H}$ :

$$
\begin{aligned}
\boldsymbol{E} & =-\nabla \phi-\frac{\partial \boldsymbol{A}}{\partial t} \\
\boldsymbol{H} & =\frac{1}{\mu_{0}} \cdot \nabla \times \boldsymbol{A}
\end{aligned}
$$

Dipole theory is use to calculate Maxwell equations above. Magnetic vector potential $\boldsymbol{A}$ can be got according to current when current component is known, Lorentz condition is expressed as below:

$$
\nabla \cdot \boldsymbol{A}+\mu_{0} \varepsilon_{0} \cdot \frac{\partial \phi}{\partial t}=0
$$

Taking (2), (3), (4) into (1), non-homogeneous general solution of (1) can be got:

$$
\left\{\begin{array}{c}
\phi(\boldsymbol{r}, t)=\frac{1}{4 \pi \varepsilon_{0}} \int_{V^{\prime}} \frac{\rho\left(\boldsymbol{r}^{\prime}, t-\frac{\left|\boldsymbol{r}-\boldsymbol{r}^{\prime}\right|}{v_{1}}\right)}{\left|\boldsymbol{r}-\boldsymbol{r}^{\prime}\right|} \cdot d V^{\prime} \\
\boldsymbol{A}(\boldsymbol{r}, t)=\frac{\mu_{0}}{4 \pi} \int_{V^{\prime}} \frac{\boldsymbol{J}\left(\boldsymbol{r}^{\prime}, t-\frac{\left|\boldsymbol{r}-\boldsymbol{r}^{\prime}\right|}{v_{1}}\right)}{\left|\boldsymbol{r}-\boldsymbol{r}^{\prime}\right|} \cdot d V^{\prime}
\end{array}\right.
$$

According to (4), we can get scalar quantity electric potential $\phi$ :

$$
\phi(\boldsymbol{r}, t)=-\frac{1}{\mu_{0} \varepsilon_{0}} \int_{-\infty}^{t} \nabla \cdot \boldsymbol{A} d t^{\prime}+\phi(t=-\infty)
$$

when $t=-\infty, \phi=0$, taking (5) into (2), E can be got:

$$
\boldsymbol{E}(\boldsymbol{r}, t)=v_{1}^{2} \int_{-\infty}^{t} \nabla(\nabla \cdot \boldsymbol{A}) d t-\frac{\partial \boldsymbol{A}}{\partial t}
$$

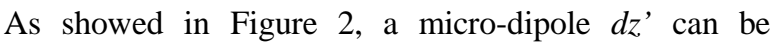
described by current $i\left(z^{\prime}, t\right), \boldsymbol{a}_{z}$ stands for unit vector potential $z$, then magnetic vector potential $\boldsymbol{A}$ can be got:

$$
d \boldsymbol{A}(\boldsymbol{r}, t)=\frac{\mu_{0}}{4 \pi} \frac{i\left(t-R / v_{1}\right)}{R} d z^{\prime} \cdot \boldsymbol{a}_{z}
$$

In the situation of dipole, the distribution between current and mirror current can be showed as the equation below:

$$
i\left(z^{\prime}, t\right)=I_{0} u\left(t-\left|z^{\prime}\right| / v\right)
$$

where $u$ is Heaviside Function.

Taking (8), (9) into (3) and (7), the electric field and magnetic field of any field point in space of cylindrical coordinate system can be got:

$$
\begin{aligned}
d E_{\mathrm{r}}(r, \boldsymbol{\phi}, z, t) & =\frac{I_{0} d z^{\prime}}{4 \pi \varepsilon_{0}}\left[\frac{3 r\left(z-z^{\prime}\right)}{R^{5}}\left(t-R / v_{1}-\left|z^{\prime}\right| / v\right) \cdot u\left(t-R / v_{1}-\left|z^{\prime}\right| / v\right)\right. \\
& \left.+\frac{3 r\left(z-z^{\prime}\right)}{c R^{4}} u\left(t-R / v_{1}-\left|z^{\prime}\right| / v\right)+\frac{r\left(z-z^{\prime}\right)}{v_{1}^{2} R^{3}} \delta\left(t-R / v_{1}-\left|z^{\prime}\right| / v\right)\right]
\end{aligned}
$$

$$
\begin{aligned}
d E_{z}(r, \boldsymbol{\phi}, z, t) & =\frac{I_{0} d z^{\prime}}{4 \pi \varepsilon_{0}}\left[\frac{2\left(z-z^{\prime}\right)^{2}-r^{2}}{R^{5}}\left(t-R / v_{1}-\left|z^{\prime}\right| / v\right) \cdot u\left(t-R / v_{1}-\left|z^{\prime}\right| / v\right)\right. \\
& \left.+\frac{2\left(z-z^{\prime}\right)^{2}-r^{2}}{v_{1} R^{4}}\left(t-R / v_{1}-\left|z^{\prime}\right| / v\right)-\frac{r^{2}}{v_{1}^{2} R^{3}} \delta\left(t-R / v_{1}-\left|z^{\prime}\right| / v\right)\right] \\
d H_{\phi}(r, \boldsymbol{\phi}, z, t)= & \frac{I_{0} d z^{\prime}}{4 \pi}\left[\frac{r}{R^{3}} u\left(t-R / v_{1}-\left|z^{\prime}\right| / v\right)+\frac{r}{v_{1} R^{2}} \delta\left(t-R / v_{1}-\left|z^{\prime}\right| / v\right)\right]
\end{aligned}
$$

where $r, z, \phi$ stand for radial coordinate, axial coordinate and azimuth respectively. 
To get the theoretical value of the lightning electromagnetic field, integral upper limit and lower limit ($h, h)$ should be determined.

According to Heaviside Function and (9), $h$ can be got from the equation below:

$$
t-\frac{\sqrt{\left(r^{2}+(z-h)^{2}\right)}}{v_{1}}=\frac{h}{v}
$$

then

so

$$
\left\{\begin{array}{c}
h=\zeta \cdot \frac{v_{1} t-\zeta z-\sqrt{\left(\zeta \cdot v_{1} t-z\right)^{2}+r^{2}\left(1-\zeta^{2}\right)}}{1-\zeta^{2}} \\
\zeta=v / v_{1}
\end{array}\right.
$$

$$
\begin{gathered}
d E_{\mathrm{r}}(r, \boldsymbol{\phi}, z, t)=\frac{d z^{\prime}}{4 \pi \varepsilon_{0}}\left[\frac{3 r\left(z-z^{\prime}\right)}{R^{5}} \int_{0}^{t} i\left(z^{\prime}, t-R / v_{1}\right) d t\right. \\
\left.+\frac{3 r\left(z-z^{\prime}\right)}{c R^{4}} i\left(z^{\prime}, t-R / v_{1}\right)+\frac{r\left(z-z^{\prime}\right)}{v_{1}^{2} R^{3}} \frac{\partial i\left(z^{\prime}, t-R / v_{1}\right)}{\partial t}\right] \\
d E_{\mathrm{z}}(r, \boldsymbol{\phi}, z, t)=\frac{d z^{\prime}}{4 \pi \varepsilon_{0}}\left[\frac{2\left(z-z^{\prime}\right)^{2}-r^{2}}{R^{5}} \int_{0}^{t} i\left(z^{\prime}, t-R / v_{1}\right) d t\right. \\
\left.+\frac{2\left(z-z^{\prime}\right)^{2}-r^{2}}{v_{1} R^{4}} i\left(z^{\prime}, t-R / v_{1}\right)-\frac{r^{2}}{v_{1}^{2} R^{3}} \frac{\partial i\left(z^{\prime}, t-R / v_{1}\right)}{\partial t}\right] \\
d B_{\phi}(r, \phi, z, t)=\frac{\mu_{0} d z^{\prime}}{4 \pi}\left[\frac{r}{R^{3}} i\left(z^{\prime}, t-R / v_{1}\right)+\frac{r}{v_{1} R^{2}} \frac{\partial i\left(z^{\prime}, t-R / v_{1}\right)}{\partial t}\right] \\
\boldsymbol{B}_{\phi}=\mu_{0} \boldsymbol{H}_{\phi}
\end{gathered}
$$

where $\mu_{0}=4 \pi \times 10^{-7} \mathrm{H} / \mathrm{m}$.

According to (15), (16), (17), (18), the lightning electromagnetic field intensity around aircraft surface can be got.

\section{INITIAL ATTACHMENT FEATURES BETWEEN LIGHTNING LEADERS AND AIRCRAFT}

The electric field intensity of any point on aircraft surface can be expressed by Gauss theorem as below:

$$
\boldsymbol{E}=\frac{\sigma}{\varepsilon} \hat{n}
$$

where $\sigma$ is the areal density of electric charge, $\varepsilon$ is the media's permittivity, $\hat{n}$ is unit normal vector of aircraft surface.

According to the points of J.C.Maxwell and M.Faraday, surface of conductor in static field is a equipotential surface, "point effect" exits on any equipotential surface and conductor surface, some scholars in China also have done some valuable researches ${ }^{[9],[10]}$, which confirmed the "point effect" that the curvature $\rho$ of any point on the conductor surface is a very important parameter to determine the electric field intensity at this point.

when $\rho>0, \sigma$ is proportional to $\rho$, that is:

$$
\sigma \propto \rho
$$

when $\rho<0, \sigma$ is directly proportional to $\rho$, that is:

$$
\begin{aligned}
& \sigma \propto 1 / \rho \\
& \rho=1 / R
\end{aligned}
$$

where $\mathrm{R}$ is spherical radius corresponding to "sharp" and "terminal" parts on conductor. From equations above, we know that when the charge distribution on conductor becomes balance, $\sigma$ is bigger at the place of the conductor surface where $\rho$ is bigger, which means there is more electric charge here. On the contrary, electric charge is fewer.

According to one-dimensional potential well model and band theory in quantum mechanics, when aircraft is in the lightning electromagnetic field environment, energy of free electric charge on the surface of conductor increases under the acting of electric force, lower energy level free charge moves to "sharp" and "terminal" parts continually in the process that lower energy level free charge moves to occupy higher energy level, consequently, areal density of electric charge at these parts increases. According to (19), electric field intensity near "sharp" and "terminal" parts increases continually, it is easy that the local strong electric field region appears, leading to electric field intensity at these "sharp" and "terminal" parts reach the breakdown electric field intensity of air, ionize air, and lightning occurs, which is showed as Figure 4.

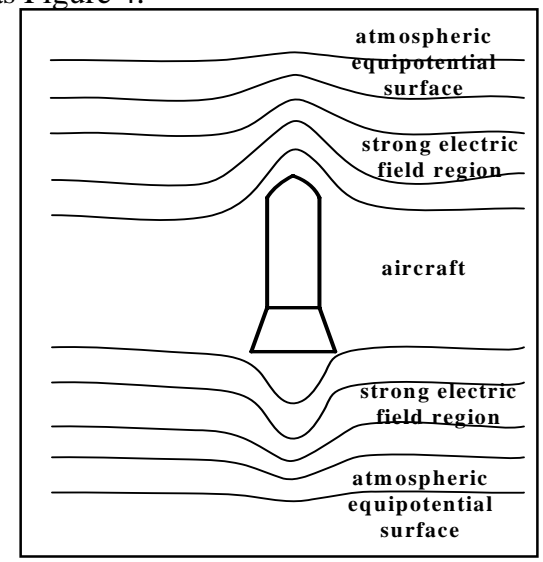

Figure 4 Local Strong Electric Field Region

\section{CHOOSING OF LIGHTNING IMPORT AND EXPORT ON AIRCRAFT}

According to the narrative above, we know that lightning occurs at the "sharp" and "terminal" parts, which means that 1A zone includes these parts, but not all of the "sharp" and "terminal” parts can become lightning import and export, different parts have different electric field intensity, only part whose electric field intensity reaches the breakdown electric field intensity of air firstly can lead lightning occur.

The relation between electric field intensity of "sharp" and "terminal" parts and electric field intensity of air can be showed as the equation below: 


$$
E_{h}=\frac{4 H / D}{\ln \frac{8 H}{D}-2} \times F
$$

where $E_{h}$ is electric field intensity of "sharp" and "terminal" parts $(\mathrm{kV} / \mathrm{m}), \mathrm{H}$ is effective conductive length of aircraft $(\mathrm{m})$, $D$ is the diameter of aircraft, F is electric field intensity of air ${ }^{[11]}$.

From (2), we know that $F$ is bigger, $E_{h}$ is bigger, it is easier to lead lightning occur. According to (15), (16), electric field intensity of field points that are closer to the lightning emitter is bigger, that is $F$ is bigger, consequently, these points include in "sharp" and "terminal" parts are most likely to become the import of lightning.

As to export, lightning leaves aircraft and transfers to the cloud under aircraft or ground, cloud under aircraft or ground can be seen as a equipotential surface, compared with big-acreage cloud or ground, electric charge on the "sharp" and "terminal" parts of aircraft can be seen as point electric charge, then electric field intensity at these points or near them can be expressed as below:

$$
E=\frac{Q}{4 \pi \varepsilon_{0} r^{3}} e_{r}
$$

where $Q$ is electric charge at "sharp" and "terminal" parts, $r$ is the distance field point away from point electric charge, $\varepsilon_{0}$ is permittivity, $e_{r}$ is direction vector.

Electric charge at each "sharp" and "terminal" part is almost at the same level, then, electric field intensity of points that are closer to equipotential surface is bigger, therefore, these points at "sharp" and "terminal" parts are most likely to become export of lightning.

\section{CONCLUSION}

Electromagnetic at any point in space, the initial attachment features and choosing of lightning import and export of aircraft are researched by the use of "point effect" theory and dipole theory in this paper, two conclusions are got through theoretical derivation:

1) Compared with other points, "sharp" and "terminal" parts are more likely to be attacked by lightning firstly. It means that these parts are include in the initial lightning attachment zone.

2) When lightning occurs, points of "sharp" and "terminal" parts which are closer to lightning emitter, cloud under aircraft or ground are most likely to become the import and export of lightning.

\section{REFERENCES}

[1] Eberle M , Marcordes R , Jaeger D , et al. Lightning protection design methodology for a very large non-rigid airship [C] . Proceedings of International Conference on Lightning and Static Electricity. [S.l.]: Society of Automotive Engineers, Inc , 2001 : 2001-01-2931.

[2] Chifford D W. Laboratory test s to determine lightning attachment points with small aircraft models[C] . Proceedings of Conference on Certification of Aircraft for Lightning and Atmospheric Electricity Hazards (Engineering Test) . Chatillon, France, 1978:9.

[3] GJB3567-99, Lightning protection qualification test techniques for military aircraft[S], 1999.

[4] SAE-ARP5146. Aircraft Lightning Test Methods[S], 2005.

[5] Bingzhong Wang. Computational Electromagnetics. Beijing: Science Press, 2002.

[6] Zhaowen Yan, Donglin Su, Xiaomei Yuan. FEKO5.4 detailed analysis and practice of electromagnetic. Beijing: China Water Power Press, 2009.

[7] Chowdhuri, P., Anderson, J.P., Chisholm, W.A., et al. Parameters of lightning strokes: A review[J]. IEEE Transactions on Power Delivery, 2005, 20(1): 346-358.

[8] Valadimir A. Rakov, Martine A. Uman. Review and evaluation of lightning return stroke models including some aspects of their application [J]. IEEE Transactions on Electromagnetic Compatibility, 1998, 40(4): 403-426.

[9] LI Rui-fang, WU Guang-ning, CAO Xiao-bin, et al. Selectivity of Spark Discharge Paths in Point/Point Gap in Air[J]. Journal of Tianjin University, 2009, 42(6):497-501.

[10] FAN Rui-ming, HU Yu-qun, QIAO Xin. Research on lightning protection of large aerobus[J]. Jiangsu Aviation, 2010, (1): 10-11.

[11] Jun Zhang. Military Meteorology[M]. Beijing: China Meteorological Press, 2005, 188-194. 